\title{
ВАВИЛОНСКАЯ БАШНЯ ПСИХОТЕРАПИИ
}

\author{
Д.А. ВАСИЛЕНКО
}

${ }^{a}$ Московский государственныий университет имени М.В. Ломоносова, 119991, Россия, Москва, Ленинские горы, 1

\begin{abstract}
Резюме
Статья посвящена теоретическому обзору направлений психотерапии и рассмотрению авторской суггестивной методики «сенсомоторного психосинтеза», особенность которой состоит в интеграции внушаемых ощущений и образов, в духе недерективного гипноза М. Эриксона и в требовании активности от пациента, в его непрекращающемся диалоге с терапевтом. Приводятся фрагменты психотерапевтической работы с пациентом по методу сенсомоторного психосинтеза. Обсуждаются различные варианты интеграции терапевтических направлений, такие как технический эклектизм, теоретическая интеграция, ассимиляционная интеграция, интеграция с опорой на общие факторы. Сенсомоторный психосинтез рассматривается как метод, позволяющий раскрывать новые возможности интеграции психотерапевтических приемов. В частности, как метод, позволяющий интергрировать психотерапевтические приемы, с учетом их отношения к уровневой организации психической деятельности; моделировать поведение человека в разных жизненных ситуациях и различные варианты реагирования в одной конкретной ситуации, включая весь спектр физиологических, чувственных, эмоциональных, поведенческих реакций. В заключении авторы присоединяются к мнению К. Юнга о том, что будущее психологии и психотерапии заключается в разработке понимания места человека в природе и смысла его существования. Отмечается, что будущее психологии не только в создании новых психопрактик, не только в их интеграции, но и в развитии их идеологического основания. Отмечается, что современная психотерапия во многом заимствовала, интегрировала и развила психотехники из различных религиозных течений. И так же как религиозные конфессии, нуждается в собственной «теологии», научно объясняющей смысл и цели человеческого существования.
\end{abstract}

Ключевые слова: психология личности, психотерапия, метод сенсомоторного психосинтеза, психосемантика, проблема смысла.

В настоящее время выделяют более 400 направлений психотерапии (поведенческую, системную семейную, когнитивную, психодинамическую, бихевиоральную и др.) (Garfield, Bergin, 1994). Однако еще с прошлого века ученые указывают на взаимодополняемость теоретических подходов, выступающих в качестве методологических основ терапевтических направлений (Birk, Brinkley-Birk, 1974). A с 1990-х гг. все большее признание приобретает комплексный подход к психотерапии (Norcross, Goldfried, 2005; Messer, 1992), где 
все большее число терапевтов предпочитают работать в рамках не одного направления, относят себя к представителям интегративного подхода (Feixas, Botella, 2004). Так, в недавнем опросе более 1000 психотерапевтов только 15\% указали, что они базировались в своей практике на теории одного направления, а в среднем один специалист в своей работе использует разработки как минимум четырех психотерапевтических направлений (Tasca et al., 2015).

Широкое распространение интегративных тенденций подтверждается тем, что в 1983 г. организовано Общество по исследованию интеграции психотерапии - международная междисциплинарная организация с постоянно растущим числом членов. Цель SEPI - содействие активному взаимодействию между терапевтами и исследователями, придерживающимися различных методологических оснований. А с 1991 г. под эгидой Американской психологической ассоциации выходит журнал «Интегративная психотерапия». Регулярно проводятся междисциплинарные конференции и обучающие и исследовательские программы, посвященные этому направлению (Norcross, Halgin, 2005; Beck et al., 2014; Boswell, Castonguay, 2007; Boswell et al., 2010; Castonguay, 2000, 2006; Lecomte et al., 1993).

Вероятно, тенденция к интеграции подходов вызвана тем, что ни одно из «чистых» направлений психотерапевтической практики не смогло стать эффективным для абсолютно всех пациентов и нозологий. Каждая из существующих психотерапевтических моделей имеет свои ограничения для тех или иных пациентов (Norcross, Goldfried, 2005). И, насколько известно, не было доказано, что какой-либо терапевтический подход эффективней, чем другие (Barth et al., 2013; Lambert, Bergin, 1992; Lambert et al., 1986; Luborsky et al., 1975; Sloane et al., 1975; Smith et al., 1980; Stiles et al., 1986).

B связи с этим большинство практикующих специалистов используют разные варианты интеграции терапевтических направлений (Zarbo et al., 2016; Feixas, Botella, 2004), такие как технический элекцизм, теоретическая интеграция, ассимиляционная интеграция, интеграция с опорой на общие факторы.

1. Технический эклекцизм (technical eclectism) - интуитивный и прагматический. Тенденцию интеграционного подхода, при котором происходит выбор терапевтических процедур вне зависимости от их «базовой» теории, называют техническим интуитивным эклекцизмом (Lazarus, Messer, 1991). При таком подходе в процессе выбора практических методик игнорируются фундаментальные методологические различия породивших их теорий. Приверженцы подобной «интеграции» снимают теоретическое бремя используемых технических приемов, опираясь лишь на предположение о том, будет ли данный прием работать с конкретным клиентом или пациентом. Зачастую терапевты, придерживающиеся данного подхода, при выборе методик опираются на интуицию, субъективную привлекательность методики и пр. Подобная хаотическая практика, несомненно, встречает серьезную критику (Eysenck, 1970).

При техническом прагматическом эклектизме критерием выбора методик выступает уровень эффективности этих методик (доказанный в эмпирических 
исследованиях) и учет особенностей пациентов (Beutler, 1992; Beutler, Clarkin, 1990; Winter, 1990, 1992).

2. Теоретическая интеграция (theoretical integration) направлена на то, чтобы объединить теоретические концепции нескольких различных психотерапевтических подходов (Stricker, Gold, 2001). Выделяют два варианта теоретической интеграции (Feixas, 1992). В гибридной теоретической интеграции объединяются теории и практики двух устоявшихся терапевтических подходов. Обычно выбираются два подхода, которые считаются взаимодополняющими, наиболее полезные теоретические и технические аспекты каждого применяются для создания общей гибридной теоретической основы (Wachtel, 1977).

Расширенная теоретическая интеграция отличается от гибридной интеграции не только тем, что включает более двух теорий, но и учетом различных аспектов функционирования человека (когнитивные, эмоциональные, поведенческие и межличностные). Такие интегрированные методы охватывают широкий спектр подходов и обогащаются вкладом различных психотерапевтических методов (Gimeno-Bayón, Rosal; 2001; Fernández-Alvarez; 2001; Dallos, 1991; Feixas et al., 1993).

3. Применяя ассимиляционную интеграцию (assimilative integration), терапевт работает в рамках одного «основного» направления, но в зависимости от динамики терапии и состояния клиента может включить в процесс терапии и методы из других психотерапевтических направлений (Stricker, Gold, 2001; Castonguay, 2011, 2013; Safran, Segal, 1990). Таким образом, специалисты получают возможность, не отказываясь от привычной теоретической базы, расширить репертуар клинических приемов и повысить эффективность своей работы. Так, например, ряд авторов описывают значимое повышение эффективности психодинамической терапии при включении в нее некоторых приемов когнитивно-поведенческой терапии (Stricker, Gold, 2003; McCullough et al., 2003; Hilsenroth, Slavin, 2008; Nelson, Castonguay, 2012). А когнитивно-поведенческая терапия повышает свою результативность при включении некоторых техник из других подходов (Blagys, Hilsenroth, 2000; Jones, Pulos, 1993; Safran, Muran, 2000; Muran et al., 2005, 2014; Constantino et al., 2008; Safran et al., 2014; Newman et al., 2008).

4. Интеграция с опорой на общие факторы (common factors). Начиная с 1930-х гг. исследователи стали обращать внимание на наличие общих, независимых от содержания и типа терапии факторов, способствующих эффективности психотерапевтических сессий. Так, еще в 1936 г. Розенцвейг высказал мнение, что такие качества психотерапевта, как способность вселять надежду и его возможность продемонстрировать клиенту альтернативное и более правдоподобное представление о себе и мире, могут быть теми факторами, которые объясняли бы эффективность психотерапии вне зависимости от применяемого подхода (Feixas, Botella, 2004). Поиск «неинструментальных», общих для всех подходов, компонентов продолжается по сей день (Norcross, Goldfried, 1992; Wampold, Imel, 2015). Со времен первых статей об общих факторах проведены десятки исследований, посвященных этой теме. На сегодняшний 
день данное направление интеграции считается самым экспериментально обоснованным (Feixas, Botella, 2004). В настоящее время к общим факторам, имеющим отношение к эффективности психотерапевтической работы, причисляют (Zarbo et al., 2016): способность терапевта внушать надежду и предоставлять альтернативное и более правдоподобное представление о себе и мире; способность давать пациентам корректирующий эмоциональный опыт (позволяющий клиенту исправить травмирующее влияние его предыдущего неудачного опыта); терапевтический альянс; ожидания позитивных изменений; такие полезные терапевтические качества специалиста, как: внимание, сочувствие, позитивное отношение и др. (Stricker, Gold, 2001; Feixas, Botella, 2004; Norcross, Goldfried, 2005; Constantino et al., 2011; Horvath et al., 2011).

Среди перечисленных общих факторов «терапевтический альянс» является наиболее экспериментально обоснованным предиктором изменений пациента (Feixas, Botella, 2004). Идея терапевтического альянса возникла в работах Фрейда (он использовал понятие «рабочий альянс»), хотя этот термин был впервые введен Гринсоном (Ibid.). Согласно его определению, терапевтический альянс состоит из способности и желания клиента работать над решением собственных проблем при помощи и активном участии терапевта. Недавний метаанализ (Horvath, Symonds, 1991) подтверждает, что именно терапевтический альянс в значительной степени связан с результатом психотерапии. Также показано, что пассивная роль клиента в процессе терапии снижает эффективность сессий (Kivlighan, 1990).

Итак, в настоящее время выделяют несколько основных направлений (с нашей точки зрения, уровней) в интегрировании психотерапевтических подходов: общих условий эффективности психотерапии (интеграция с опорой на общие факторы), различных способов компиляции и встраивания технических приемов (техническая и ассимиляционная интеграция), попыток организовать площадку для объединения теоретических концепций различных психотерапевтических подходов с гипотетической возможностью разработки «Великой единой теории» психотерапии (Stricker, Gold, 2001).

Авторы сходятся во мнении, что интегративная психотерапия эффективна в качестве метода терапии при таких психических расстройствах, как депрессии различного генеза, тревожные расстройства, диссоциативные и другие растройства личности и т.д. (Reay et al., 2003; Kellett, 2005; Hamidpour et al., 2011; Stangier et al., 2011; Masley et al., 2012; Roediger, Dieckmann, 2012; Clarke et al., 2013; Miniati et al., 2014); интегративный подход позволяет лучше адаптировать терапию к особенностям и потребностям каждого клиента; в рамках интегративной психотерапии техники не применяются к пассивному клиенту, клиент рассматривается как активный участник сессий, в ходе которых терапевт представляет возможные альтернативные варианты ви́дения себя или ситуаций, позволяющие скорректировать негативный опыт клиента.

Однако теоретический анализ проблемы невольно вызывает ощущение, что каждое направление психотерапии и каждый прием имеет своего рода «радиус действия», т.е. позволяет корректировать лишь часть психологических феноменов; и на привычном пятачке «чистого» направления специалисты 
имеют ориентировочную основу действия в виде реперных точек методологических оснований своего базового направления (или нескольких направлений), но сталкиваются со «слепыми зонами» его возможностей. А чтобы попасть в другую область облака психологических феноменов клиента, специалистам приходится использовать другой теоретический подход или технический прием, часто вслепую, ориентируясь на разрозненную россыпь эмпирически установленных фактов или на островки понимания разрозненных теорий нескольких направлений. И, к сожалению, результат часто напоминает неполную мозаику, а процесс - нащупывание направления пути без четко простроенной карты местности.

Таким образом, проблемное поле интеграции психотерапевтических направлений находится в процессе противоречивого становления и провоцирует множество методологических дискуссий относительно вариантов структурирования и систематизации теоретических интерпретаций психологических феноменов - объектов психотерапии, а также способов их произвольной регуляции. Под разными названиями в россыпи психотехник часто скрываются приемы, реализующие действие одного и того же психологического механизма («лечение сказкой» в сказкотерапии, «терапевтические истории» Милтона Эриксона или Притчи Иисуса Христа в Евангелиях, если рассматривать психотерапевтический аспект религиозных практик).

На данном этапе развития интегративной психотерапии нам представляется важным поиск «катализатора», позволяющего включить в единую «реакцию» различные направления психотерапевтической практики, а также определение системы «каскада реакций» для получения «единого терапевтического сплава», обладающего достаточной прочностью и гибкостью.

Используя аналогию с катализатором, мы особое внимание обращаем на то, что интеграция психотерапевтических приемов должна не только эксплуатировать изначальный терапевтический эффект психотехник, но и раскрывать новые возможности и свойства приемов, снимая присущие им ограничения; при этом сохраняя или создавая условия (в случае отсутствия таковых в изначальном приеме) для формирования активности субъекта в психотерапевтической деятельности. В конце 1980-х гг. на факультете психологии МГУ была предложена уникальная методика, отвечающая данным условиям. Она включает множество авторских приемов, разработанных В.В. Кучеренко в ходе многолетней психотерапевтической практики, совместных экспериментальных исследований измененных состояний сознания, выполненных В.В. Кучеренко и В.Ф. Петренко, теоретически осмыслена и получила название сенсомоторного психосинтеза (Петренко, Кучеренко, 2002, 2019).

Сенсомоторный психосинтез - методика, включающая специально организованную последовательность тестовых заданий, предполагающих концентрацию внимания на тех ощущениях, представлениях, которые возникают при их решении. В результате поэтапного выполнения данных заданий у субъекта формируется целостный образ иллюзорной действительности. Причем данная методика позволяет произвольно регулировать перцептивные характеристики моделируемого образа. Интеграция ощущений различных модальностей в 
единый мультимодальный образ происходит в процессе диалога клиента и психолога. В ходе такого диалога специалист имеет возможность получать информацию о состоянии и переживаниях субъекта, таким образом оценивать качество решения текущих тестовых заданий, корректировать ход решения, подбирать следующие задачи с учетом индивидуальных особенностей клиента. А клиент, в свою очередь, получает информацию от психолога о причинах неудач и путях преодоления ошибок.

В процессе диалога, организованного таким образом, субъект приобретает возможность стать наблюдателем, а затем при помощи психолога - творцом внутренних процессов фантазии. Фиксация внимания на внутренних процессах (внутренняя позиция субъекта) способствует индукции особых измененных состояний сознания субъекта. В таком особом, трансовом состоянии чувственная ткань сознания выходит на первый план, а субъект погружается в конструируемые миры наглядно чувственных переживаний. «Здесь же все приобретает реальность. Представления приобретают яркость видений и живо переживаются, ощущение собственного движения и другие ощущения (тактильные, вкусовые, обонятельные - каждое) вносят свой собственный эмоциональный оттенок в картину, открывающуюся субъекту, снимая тем самым элемент условности с переживаемых событий... субъект сохраняет чувство самотождественности и произвольности собственной активности - ему никто ничего не навязывает; его Я активно. "Миры”, возникающие в сознании субъекта, суть продукты сотрудничества, взаимодействия его и психолога» (Кучеренко, 2010, с. 65). Именно эти возможности измененных состояний сознания, активизируемые при помощи сенсомоторного психосинтеза, и могут быть фундаментом для реальной интеграции психотерапевтических приемов.

Авторы анализируют приемы работы с процессом внутренней коммуникации между различными «частями» Я в гештальттерапии, в психосинтезе Ассаджиоли; переключения эго-состояний («Родитель», «Взрослый», «Ребенок») в трансактном анализе Эрика Берна; смену ролей клиента и властвующего над ним «монстра» в процессуально ориентированной психотерапии; разыгрывание различных ситуаций в бихевиоральной терапии и пр. Они отмечают: «Все эти техники, затрагивая те или иные структуры личности, сохраняют момент игровой отстраненности, драматизации, а следовательно, и определенной дистанции до ядерного переживания Я... Сенсомоторный психосинтез, как представляется, снимает присущие этим техникам ограничения, предоставляя режим наибольшего благоприятствования для достижения тех эффектов, на которые они имели бы основания притязать» (Там же, с. 64). Сенсомоторный психосинтез позволяет моделировать, с одной стороны, поведение человека в различных жизненных ситуациях (Петренко, Кучеренко, 2019), с другой стороны, различные варианты чувствования, реагирования субъекта (структур Я) в одной и той же ситуации (Нуркова, Василенко, 2013). Причем не игровое моделирование, а проживание полного спектра чувственных и эмоциональных переживаний. Этот метод позволяет воспроизводить 
«выпадающие» звенья, недоступные по той или иной причине для спонтанной активизации пациентам.

Так, в примере при работе с анорексией мы заметили, что у пациентов легко воспроизводятся мультимодальные образы эмоционально нейтральных ситуаций, не связанных с темой употребления пищи. Например, пациентка, страдающая анорексией, описывает образ горной местности, сконструированный в ходе сеанса:

Психолог (П): Оглянитесь, вершины заснежены?

Клиент (К): Да, наверху да. Высоко... Снег яркий... белый-белый.

П: Снег искрится?

$K:$ да, солнце яркое.

П: Щеки уже припекает?

К: Ой! Щеки горячие.

П: Посмотрите, как снежинки ветерок поднимает! Обратите внимание на ощущение в области лба, висков, вы заметите, какой ветер холодный.

$K$ : Нос холодный... лицо щиплет... Снежинка красивая (рассматривает ладонь)... пальцы колет.

П: Тихо вокруг?

К: ... Эхо... лавина...

П: Где?

$K$ : Вон (показывает рукой)... Пахнет... зимой (глубоко вдыхает)... Хорошо, как (улыбается).

Однако при моделировании ситуации, связанной с употребление пищи (ресторан), образ становится неустойчивым, диссоциированным, мономодальным. Пациентке удается увидеть только «маленькие картинки», неустойчивые расплывающиеся образы-вспышки (гардероб в ресторане, цвет скатерти, пианино рядом со столиком). Любые попытки активизировать кинестетические, ольфакторные, аудиальные ощущения вызывают мгновенное выпадение из текущей деятельности и отвлечение на малозначимые детали окружающей действительности (отмечает, что ковер в кабинете у психолога «хороший»). «Внутренний запрет» на употребление пищи и любые ощущения, связанные с ней, удается убрать благодаря ряду приемов, реализуемых при помощи сенсомоторного психосинтеза. Одним из таких приемов, позволяющих восстановить «неработающие звенья» кинестетических, ольфакторных, аудиальных ощущений, связанных с едой, стал прием ассоциации с субъектом, получающим удовольствие от употребления пищи. Так, наша пациентка в прямом смысле слова побывала в шкуре кота, гуляющего по рынку. Она прочувствовала, как пружинят ее лапы, когда она гуляет под прилавками. Видела цвет своих лап, а позже, когда заглянула в лужу, рассмотрела «свою» мордочку. Поточила когти о бревна на строительных рядах, прочувствовала запах масляных красок, услышала рев бензопилы. После этого нам удалось встраивать в эмоционально нейтральную иллюзорную мультимодальную действительность «недостающие элементы». Так, гуляя по строительным рядам, она заметила по соседству какие-то яркие коробочки, в них «лапы приятно проваливаются». Оказалось, что эти коробочки - со специями. Проживая эти зрительные, 
кинестетические и аудиальные ощущения, пациентка вдруг начала чувствовать запахи корицы и перца. Впоследствии ей удалось прогуляться и в молочный отдел, где у нее «в углу стоит миска со сметаной», и поохотиться на небрежно лежащую сосиску в мясном ряду. Помимо всего прочего, ассоциация с этим образом помогла прочувствовать и ощущение голода, и ощущение приятной сытости, и, что не менее важно, новую для нее схему тела. Таким образом, благодаря сенсомоторному психосинтезу становится доступна работа с «механизмами» разного уровня: физиологическими, эмоциональными, поведенческими и т.д.

Говоря о процессах внутренней коммуникации субъекта, структурах Я, поведенческих и эмоциональных реакциях на ту или иную ситуацию и т.д., мы обращаемся к сложной, высокоорганизованной психической деятельности. Именно она в подавляющем большинстве случаев является предметом психотерапевтической работы. Как известно (П.К. Анохин, Н.А. Бернштейн, А.Р. Лурия), психические процессы человека являются сложными функциональными системами. Любой вид психической деятельности осуществляется благодаря работе трех основных функциональных блоков (Лурия, 2006): 1) блок, обеспечивающий регуляцию тонуса и бодрствования; 2) блок получения, переработки и хранения информации, поступающей из внешнего мира; 3) блок программирования, регуляции и контроля психической деятельности. «Каждая форма сознательной деятельности всегда является сложной функциональной системой и осуществляется, опираясь на совместную работу всех трех блоков мозга, каждый из которых вносит свой вклад в осуществление психического процесса в целом» (Там же, с. 126).

Возвращаясь к теме психотерапевтической интеграции и вопросу рассмотрения системы «каскада реакций» (последовательности, структуры интеграции различных терапевтических направлений и их приемов), нам представляется, что анализ предмета психотерапевтических воздействий того или иного направления с позиции соотношения его с уровневой организацией психической деятельности, в частности с тремя функциональными блоками Лурии, может быть весьма актуальным. Так, например, рассмотренная выше работа механизмов внутренней коммуникации между «частями» Я, на наш взгляд, имеет большее соотношение с вкладом 2-го и 3-го блоков, а методика биологически обратной связи с большим вкладом активности - 1-го и 2-го блоков. Таким образом, «вертикальная» системная организация мозговых структур может быть рассмотрена в качестве ориентира для «направлений сборки» психотерапевтических приемов в рамках терапевтических сессий.

Обращает на себя внимание ограниченность подавляющего большинства терапевтических направлений в возможности восстановления работы функций в звеньях первого и отчасти второго блока у лиц, обратившихся за психотерапевтической помощью и не имеющих ярко выраженной нейропсихологической симптоматики. Однако, как показывает практика, восстановление функций в звеньях второго и первого блока, как правило, существенно ускоряет возвращение навыков целеполагания, целедостижения, регуляции собственного поведения и эмоционального состояния, социальных взаимоотношений; 
навыков, сбои в работе которых чаще всего озвучиваются клиентами как причина обращения к психотерапевту. Существенные наработки в области моделирования физиологических состояний (первый блок мозга имеет связь с обменными процессами в организме, с «внутренним хозяйством организма»Лурия, 2006) излагаются в публикациях российских врачей и физиологов (Буль, 2015; Гримак, 2009; Платонов, 1957). Однако методики, применяемые этими авторами, имеют одно существенное ограничение - данные методики применяются только к гипнабельным пациентам, т.е. могут способствовать восстановлению лишь небольшому количеству пациентов, обратившихся за помощью.

В рамках изучения работы автобиографической памяти также предприняты попытки исследования влияния актуализации в памяти воспоминаний определенного содержания на физиологическое состояние испытуемых. К. Пездек и Р. Салим установили, что испытуемые, актуализировавшие воспоминания об успешном опыте публичных выступлений в раннем детстве, эффективнее выступали на публике, имели более низкий показатель тревожности, а также уровень кортизола в слюне у них был ниже, чем у испытуемых в контрольной группе (Pezdek, Salim, 2011). Авторы дали участникам экспериментальной группы фактически суггестивную инструкцию, утверждающую, что у данного конкретного испытуемого в возрасте до 10 лет имелся позитивный опыт публичных выступлений. Испытуемым давали 5 минут, чтобы вспомнить это событие, подумать и описать его. Фактически актуализировали трансовое состояние у испытуемых с последующей просьбой описать параметры спонтанно возникшего перцептивного образа, таким образом обходя проблему гипнабельности испытуемых, но не контролируя параметры актуализируемого образа (ассоциированность, мультимодальность, эмоциональную включенность и т.д.). Преимущество моделирования состояний при помощи методики сенсомоторного психосинтеза состоит в том, что позволяет формировать ассоциированные мультимодальные образы с заданными перцептивными характеристиками. И в отличие от классического медицинского гипноза, эксплуатирующего гипнабельность субъекта, сенсомоторный психосинтез не зависит от этого фактора и позволяет реконструировать физиологические реакции и психические образы вне зависимости от степени гипнабельности клиента.

Нам не удалось найти ни одного направления психотерапевтической практики, формирующей переключаемость состояний общей активности и расслабления. Существует множество приемов в различных терапевтических подходах, восстанавливающих способность клиента актуализировать то или иное функциональное состояние (релаксации, медитации, аутогенные тренировки), но не их переключаемость. Сенсомоторный психосинтез направлен на формирование как каталептических, так и релаксационных состояний, а также ставит своей целью восстановление их переключаемости (Петренко, Кучеренко, 2019). Восстановление переключаемости каталептических и релаксационных состояний, а также поочередное воспроизведение с переключением симпатических и парасимпатических эффектов - приемы, на наш взгляд, способствующие оптимизации работы первого блока мозга и вегетативной 
нервной системы. Данное положение проверено нами в ходе многолетней практической деятельности и требует дальнейшего теоретического и экспериментального развития.

\section{Заключение}

Как полагал Карл Юнг, будущее психологии - в первую очередь не в терапии, а скорее в едином понимании природы и места человечества в ней (Линдорф, 2013, с. 121). Речь идет, таким образом, не только о создании новых психологических практик, но и о развитии некоторого идеологического основания этих практик. Или, как об этом говорят в религиозных конфессиях, создания некоей телеологии, дающей и определяющей систему ценности данной конфессии. Мировые религии, помимо своеобразной психотерапии, призванной примирить человека с миром и с Богом, обладают сакральными текстами, объясняющими мироустройство. Между мировыми религиями много общего в трактовке мироустройства, но много и различий. Например, самая ранняя из мировых религий - буддизм - характеризует бытие как мир страдания. И буддийские психопрактики, такие как медитация или ретрит, ведут к угасанию влечений и желаний (отрубанию корня страдания) и в конечном итоге - к паранирване как добровольному уходу из мира страданий. Или же, достигнув просветления, принятию роли бодхисатвы, из сострадания ко всем живым существам оставшегося в этом мире для проповедования дхармы как пути преодоления страдания. Христианство проповедует любовь к ближнему через любовь к Богу, создавшему этот мир, принявшему мученическую смерть («смерть смертью поправ») и доказавшему через свое воскресение ее несуществование и возможность воскресения из мертвых для всех живущих. Введя идею Страшного Суда и последующего воскресения из мертвых, авраамические религии (иудаизм, христианство и ислам) ввели в миропонимание вектор спасения и будущей благодати в раю. На религиозной основе православия мыслится и построение вертикали психотерапевтической практики (Василюк, 2004; Слободчиков, 2004).

Современная психотерапия, помимо развития психопрактик и психотехник (психоанализ, сенсомоторный психосинтез, гипноз или холотропное дыхание, во многом заимствованных из практики йоги, иудаизма, буддизма, индуизма, практики созерцания в христианском исихазме, зикра и танца дервишей в исламе и т.п.), нуждается в собственной теологии, научно объясняющей смысл и цели человеческого существования. В наших работах по методологии психосемантики мы пытаемся создать такую «научную теологию» в контексте взаимодействия сознания и бессознательного как проводника космического бессознательного, рассматривая человеческую эволюцию как звено в глобальной космической эволюции (Назаретян, 2001, 2013; Менский, 2011; Петренко, 2018, 2019; Петренко, Супрун, 2017), и, соответственно, понять смысл человеческого существования в контексте целенаправленной эволюции Духа. 


\section{Литература}

Буль, П. И. (2015). Гипноз в клинике внутренних болезней: Опыт психотерапии - гипноза и внушения в клинике (3-е изд.). М.: ЛЕНАНД.

Василюк, Ф. Е. (2004). Исповедь и психотерапия. Московский психотерапевтический журнал. Специальный выпуск по христианской психологии, 4, 79-90.

Гримак, Л. П. (2009). Моделирование состояний человека в гипнозе. М.: Книжный дом «Либроком».

Кучеренко, В. В. (2010). Процессы категоризации в измененных состояниях сознания (Кандидатская диссертация). Московский государственный университет им. М.В. Ломоносова.

Линдорф, Д. (2013). Юнг и Паули: встреча двух великих умов. М.: Клуб Касталия.

Лурия, А. Р. (2006). Основы нейропсихологии. М.: Академия.

Менский, М. Б. (2011). Сознание и квантовая механика (жизнь в параллельных мирах). Фрязино: Век 2.

Назаретян, А. П. (2001). Цивилизационные кризисы в контексте универсальной истории. М.: ПЕР СЭ. Назаретян, А. П. (2013). Нелинейное будущее. М.: МБА.

Нуркова, В. В., Василенко, Д. А. (2013). Формирование вариативного репертуара самоопределяющих воспоминаний как средство развития самоидентичности. Вестник РГГУ. Серия Психологические науки, 18(119), 11-30.

Петренко, В. Ф. (2018). Космические истоки религиозного чувства. Методология и история психологии, 3, 5-27.

Петренко, В. Ф. (2019). Одни ли мы во Вселенной или возможна встреча с иными цивилизациями. Методология современной психологии, 9, 245-259.

Петренко, В. Ф., Кучеренко, В. В. (2002). Искусство суггестивного воздействия. В кн. Российская наука: дорога жизни (с. 350-357). М.: Октопус/Журнал «Природа».

Петренко, В. Ф., Кучеренко, В. В. (2019). Теория и практика сенсомоторного психосинтеза. Вестник Российской академии наук, 89(2), 147-156.

Петренко, В. Ф., Супрун, А. П. (2017). Методологические пересечения психосемантики сознания и квантовой физики. М.: Нестор-История.

Платонов, К. И. (1957). Слово как физиологический и лечебный фактор (2-е изд., заново пераб. и доп.). М.: Медгиз.

Слободчиков, В. И. (2004). О перспективах построения христиански ориентированной психологии. Московский психотерапевтический журнал. Специальный выпуск по христианской психологии, 4, 5-18.

Ссылки на зарубежные источники см. в разделе References после англоязычного блока.

Василенко Дарья Александровна - практикующий психотерапевт, кандидат психологических наук.

Сфера научных интересов: психология личности, психотерапия, измененные состояния сознания.

Контакты: Baskun4ak@mail.ru 
Кучеренко Владимир Вилетарьевич - старший научных сотрудник, факультет психологии, МГУ имени М.В. Ломоносова, кандидат психологических наук.

Сфера научных интересов: психология личности, гипнотерапия.

Контакты: vvkucherenko@gmail.com

Петренко Виктор Федорович - заведующий лабораторией «Общение и психосемантика», факультет психологии, МГУ имени М.В. Ломоносова, доктор психологических наук, членкорреспондент РАН, профессор МГУ, член-корреспондент РАН, профессор МГУ.

Сфера научных интересов: общая психология, психосемантика, теория сознания и бессознательного.

Контакты: victor-petrenko@mail.ru

\title{
The Tower of Babel of Psychotherapy
}

\author{
D.A. Vasilenko ${ }^{a}$, V.V. Kucherenko ${ }^{a}$, V.F. Petrenko ${ }^{a}$
}

${ }^{a}$ Lomonosov Moscow State University, 1 Leninskie Gory, Moscow, 119991, Russian Federation

\begin{abstract}
The article is devoted to a theoretical review of psychotherapy directions and description of the authors' suggestive "sensorimotor psychosynthesis" technique, which specifics lies in the integration of suggested feelings and images, in the style of M. Erickson's non-directive hypnosis, that requires activity from the patient in his ongoing dialogue with the therapist. Fragments of psychotherapeutic work with the patient according to the method of sensorimotor psychosynthesis are given. Various options for integrating therapeutic areas such as technical eclecticism, theoretical integration, assimilative integration and integration based on common factors are discussed. Sensorimotor Psychosynthesis is considered as a method that allows to reveal new possibilities for the integration of psychotherapeutic techniques. In particular, it is argued to be a method for integrating psychotherapeutic techniques, taking into account their relationship to the level organization of mental activity; to model human behavior in different life situations and various response options in one specific situation, including the whole spectrum of physiological, sensory, emotional and behavioral reactions. In conclusion, the authors support C. Jung's opinion that the future of psychology and psychotherapy is to develop an understanding of human's place in nature and the meaning of his existence. It is noted that the future of psychology is not only in the creation of new psycho-practices, not only in their integration, but also in the development of their ideological foundation. It is noted that modern psychotherapy has largely borrowed, integrated and developed psychotechnics from various religious movements. And just as religious denominations need their own "theology", scientifically explaining the meaning and purpose of human existence.
\end{abstract}

Keywords: personality psychology, psychotherapy, sensorimotor psychosynthesis, psychosemantics, the problem of meaning. 


\section{References}

Barth, J., Munder, T., Gerger, H., Nьesch, E., Trelle, S., Znoj, H., ... Cuijpers, P. (2013). Comparative efficacy of seven psychotherapeutic interventions for patients with depression: a network metaanalysis. PLoS Medicine, 10, e1001454. doi:10.1371/journal.pmed.1001454

Beck, J. G., Castonguay, L. G., Chronis-Tuscano, A., Klonsky, E. D., McGinn, L. K., \& Youngstrom, E. A. (2014). Principles for training in evidence-based psychology: Recommendations for the graduate curricula in clinical psychology. Clinical Psychology: Science and Practice, 21, 410-424. doi:10.1111/cpsp.12079

Beutler, L. E. (1992). La situaciyn actual y las contribuciones de la investigaciyn en psicoterapia [The present situation and the contributions of psychotherapy research]. Revista Argentina de Clínica Psicolygica, 1, 203-228. (in Spanish)

Beutler, L. E., \& Clarkin, J. F. (1990). Systematic treatment selection: Toward targeted therapeutic interventions. New York: Brunner/Mazel.

Birk, L., \& Brinkley-Birk, A. W. (1974). Psychoanalysis and behavior therapy. The American Journal of Psychiatry, 131, 499-510.

Blagys, M. D., \& Hilsenroth, M. J. (2000). Distinctive feature of short-term psychodynamic-interpersonal psychotherapy: A review of the comparative psychotherapy process literature. Clinical Psychology: Science and Practice, 7, 167-188. doi:10.1093/clipsy/7.2.167

Boswell, J. F., \& Castonguay, L. G. (2007). Psychotherapy training: Suggestions for core ingredients and future research. Psychotherapy, 44, 378-383. doi:10.1037/0033-3204.44.4.378

Boswell, J. F., Nelson, D. L., Nordberg, S. S., McAleavey, A. A., \& Castonguay, L. G. (2010). Competency in integrative psychotherapy: Perspectives on training and supervision. Psychotherapy, 47, 3-11. doi:10.1037/a0018848

Bul', P. I. (2015). Gipnoz v klinike vnutrennikh boleznei: Opyt psikhoterapii - gipnoza i vnusheniya v klinike [Hypnosis in internal medicine clinic: Experience of psychotherapy - hypnosis and suggestion in clinic] (3rd ed.). Moscow: LENAND. (in Russian)

Castonguay, L. G. (2000). A common factor approach to psychotherapy training. Journal of Psychotherapy Integration, 10, 263-282. doi:10.1023/A.1009496929012

Castonguay, L. G. (2006). Personal pathways in psychotherapy integration. Journal of Psychotherapy Integration, 16, 36-58. doi:10.1037/1053-0479.16.1.36

Castonguay, L. G. (2011). Psychotherapy, psychopathology, research and practice: Pathways of connections and integration. Psychotherapy Research, 21, 125-140. doi:10.1080/ 10503307.2011.563250

Castonguay, L. G. (2013). Psychotherapy outcome: An issue worth re-visiting 50 years later. Psychotherapy, 50, 52-67. doi:10.1037/a0030898

Clarke, S., Thomas, P., \& James, K. (2013). Cognitive analytic therapy for personality disorder: randomised controlled trial. The British Journal of Psychiatry, 202(2), 129-134. doi:10.1192/bjp.bp.112.108670

Constantino, M. J., Arnkoff, D. B., Glass, C. R., Ametrano, R. M., \& Smith, J. Z. (2011). Expectations. Journal of Clinical Psychologe, 67(2), 184-192. doi:10.1002/jclp.20754

Constantino, M. J., Marnell, M. E., Haile, A. J., Kanther-Sista, S. N., Wolman, K., Zappert, L., \& Arnow, B. A. (2008). Integrative cognitive therapy for depression: A randomized pilot comparison. Psychotherapy, 45, 122-134. doi:10.1037/ 0033-3204.45.2.122

Dallos, R. (1991). Family belief systems: Therapy and change. Buckingham, UK: Open University Press. 
Eysenck, H. J. (1970). A mishmash of theories. International Journal of Psychiatry, 9, 140-146.

Feixas, G. (1992). Constructivismo e integraciyn en psicoterapia [Constructivism and integration in psychotherapy]. Revista de Psicoterapia, 3(12), 101-108. (in Spanish)

Feixas, G., \& Botella, L. (2004). Psychotherapy integration: reflections and contributions from a constructivist epistemology. Journal of Psychotherapy Integration, 14(2), 192-222. doi:10.1037/10530479.14.2.192

Feixas, G., Procter, H., \& Neimeyer, G. (1993). Convergent lines of assessment: Systemic and constructivist contributions. In G. J. Neimeyer (Ed.), Casebook in constructivist assessment (pp. 143-178). London: Sage.

Fernández-Alvarez, H. (2001). Fundamentals of an integrated model of psychotherapy. Northvale, NJ: Jason Aronson.

Garfield, S., \& Bergin, A. (1994). Introduction and historical overview. In A. Bergin \& S. Garfield (Eds.), Handbook of psychotherapy and behaviour change (pp. 3-18). Chichester: Wiley.

Gimeno-Bayón, A., \& Rosal, R. (2001). Psicoterapia integradora humanista. Manual para el tratamiento de 33 problemas psicosensoriales, cognitivos y emocionales [Humanistic integrative psychotherapy: A handbook for the treatment of 33 psychosensory, cognitive, and emotional problems]. Bilbao, Spain: Desclée de Brouwer. (in Spanish)

Grimak, L. P. (2009). Modelirovanie sostoyanii cheloveka v gipnoze [Modeling of human states in hypnosis]. Moscow: Knizhnyi dom "Librokom".

Hamidpour, H., Dolatshai, B., Shahbaz, A. P., \& Dadkhah, A. (2011). The efficacy of schema therapy in treating women's generalized anxiety disorder. Iranian Journal of Psychiatry and Clinical Psychology, 16(4), 420-431.

Hilsenroth, M. J., \& Slavin, J. M. (2008). Integrative dynamic treatment for comorbid depression and borderline conditions. Journal of Psychotherapy Integration, 18, 377-409. doi:10.1037/ a0014317

Horvath, A. O., Del Re, A. C., Flückiger, C., \& Symonds, D. (2011). Alliance in individual psychotherapy. Psychotherapy (Chicago, IL), 48, 9-16. doi:10.1037/a0022186

Horvath, A. O., \& Symonds, B. D. (1991). Relation between alliance and outcome in psychotherapy: A meta-analysis. Journal of Counseling Psychology, 38, 139-149.

Jones, E. E., \& Pulos, S. M. (1993). Comparing the process in psychodynamic and cognitive-behavioral therapies. Journal of Consulting and Clinical Psychology, 61, 306-316. doi:10.1037/ 0022006X.61.2.306

Kellett, S. (2005). The treatment of dissociative identity disorder with cognitive analytic therapy: experimental evidence of sudden gains. Journal of Trauma and Dissociation, 6(3), 55-81. doi:10.1300/J229v06n03_03

Kivlighan, J. (1990). Therapeutic alliance and client active role in psychotherapy. Psychological Medicine, 11, 535-550.

Kucherenko, V. V. (2010). Protsessy kategorizatsii v izmenennykh sostoyaniyakh soznaniya [Processes of categorization in the altered states of consciousness] (PhD dissertation). Lomonosov Moscow State University, Moscow, Russian Federation. (in Russian)

Lambert, M. J., \& Bergin, A. E. (1992). Achievements and limitations of psychotherapy research. In D. K. Freedheim (Ed.), History of psychotherapy: A century of change (pp. 360-390). Washington, DC: American Psychological Association.

Lambert, M. J., Shapiro, D. A., \& Bergin, A. E. (1986). The effectiveness of psychotherapy. In S. L. Garfield \& A. E. Bergin (Eds.), Handbook of psychotherapy and behavior change (3rd ed., pp. 157212). New York: Wiley. 
Lazarus, A. A., \& Messer, S. B. (1991). Does chaos prevail? An exchange on technical eclecticism and assimilative integration. Journal of Psychotherapy Integration, 2, 143-158

Lecomte, C., Castonguay, L. G., Cyr, M., \& Sabourin, S. (1993). Supervision and instruction in doctoral psychotherapy integration. In G. Stricker \& J. R. Gold (Eds.), Comprehensive handbook of psychotherapy integration (pp. 483-498). New York: Plenum.

Lindorff, D. (2013). Yung i Pauli: vstrecha dvukh velikikh umov [Jung and Pauli: The meeting of two great minds]. Moscow: Klub Kastaliya. (in Russian; transl. of: Lindorff, D. (2004). Pauli and Jung: The meeting of two great minds. Wheaton, IL: Quest Books.)

Luborsky, L., Singer, B., \& Luborsky, L. (1975). Comparative studies of psychotherapies. Archives of General Psychiatry, 32, 995-1008.

Luria, A. R. (2006). Osnovy neiropsikhologii [Fundamentals of neuropsychology]. Moscow: Akademiya. (in Russian)

Masley, S. A., Gillanders, D. T., Simpson, S. G., \& Taylor, M. A. (2012). A systematic review of the evidence base for Schema Therapy. Cognitive Behaviour Therapy, 41, 185-202. doi:10.1080/16506073.2011.614274

McCullough, L., Kuhn, N., Andrews, S., Kaplan, A., Wolf, J., \& Lanza Hurley, C. (2003). Treating affect phobia: A manual for short-term dynamic psychotherapy. New York: Guilford Press.

Menskii, M. B. (2011). Soznanie i kvantovaya mekhanika (zhizn'v parallel'nykh mirakh) [Consciousness and quantum mechanics (life in parallel worlds)]. Fryazino: Vek 2. (in Russian)

Messer, S. B. (1992). A critical examination of belief structures in integrative and eclectic psychotherapy. In J. C. Norcross \& M. R. Goldfried (Eds.), Handbook of psychotherapy integration (pp. 130-165). New York: Basic Books.

Miniati, M., Callari, A., Calugi, S., Rucci, P., Savino, M., Mauri, M., \& Dell'Osso, L. (2014). Interpersonal psychotherapy for postpartum depression: a systematic review. Archives of Women's Mental Health, 17, 257-268. doi:10.1007/s00737-014-0442-7

Muran, J. C., Safran, J. D., Eubanks-Carter, C., Gorman, B. S., \& Winston, A. (June, 2014). Exploring changes in interpersonal process, intermediate and ultimate outcome in a within-subject experimental study of an alliance-focused training. Paper presented at the annual meeting of the Society for Psychotherapy Research, Copenhagen, Denmark.

Muran, J. C., Safran, J. D., Samstag, L. W., \& Winston, A. (2005). Evaluating an alliance-focused treatment for personality disorders. Psychotherapy: Theory, Research, Practice, Training, 42, 532-545. doi:10.1037/0033-3204.42.4.532

Nazaretyan, A. P. (2001). Tsivilizatsionnye krizisy v kontekste universal'noi istorii [Civilizational crises in the context of universal history]. Moscow: PER SE. (in Russian)

Nazaretyan, A. P. (2013). Nelineinoe budushchee [Non-linear future]. Moscow: MBA. (in Russian)

Nelson, D. L., \& Castonguay, L. G. (2012). The systematic use of homework in psychodynamic Interpersonal psychotherapy for depression. Paper presented at the annual meeting of the Society for Psychotherapy Research, Virginia Beach, VA.

Newman, M. G., Castonguay, L. G., Borkovec, T. D., Fisher, A. J., \& Nordberg, S. S. (2008). An open trial of integrative therapy for generalized anxiety disorder. Psychotherapy: Theory, Research, Practice, Training, 45, 135-147. doi:10.1037/0033-3204.45.2.135

Norcross, J. C., \& Goldfried, M. R. (1992). Handbook of psychotherapy integration. New York: Basic Books.

Norcross, J. C., \& Goldfried, M. R. (2005). Handbook of psychotherapy integration (2nd ed.). Oxford: Oxford University Press. 
Norcross, J. C., \& Halgin, R. P. (2005). Training in psychotherapy integration. In J. C. Norcross \& M. R. Goldfried (Eds.), Handbook of psychotherapy integration (2nd ed., pp. 439-458). New York: Oxford University Press.

Nourkova, V. V., \& Vasilenko, D. A. (2013). Forming of variative repertory of self-defining recollections as mean for developing of self-identity. RSUH/RGGU Bulletin. Series Psychological Studies, 18(119), 11-30. (in Russian)

Petrenko, V. F. (2018). The cosmic origins of religious feeling. Metodologiya i Istoriya Psikhologii [Methodology and History of Psychology], 3, 5-27. (in Russian)

Petrenko, V. F. (2019). Odni li my vo Vselennoi ili vozmozhna vstrecha s inymi tsivilizatsiyami [Are we alone in the Universe, or there is a possibility of meeting with other civilizations?]. Metodologiya Sovremennoi Psikhologii, 9, 245-259. (in Russian)

Petrenko, V. F., \& Kucherenko, V. V. (2002). Iskusstvo suggestivnogo vozdeistviya [The art of suggestive influence]. In Rossiiskaya nauka: doroga zhizni [Russian science: A road of life] (pp. 350-357). Moscow: Oktopus/Priroda. (in Russian)

Petrenko, V. F., \& Kucherenko, V. V. (2019). Theory and practice of sensori-motor psychosynthesis. Vestnik Rossiiskoi Akademii Nauk, 89(2), 147-156. (in Russian)

Petrenko, V. F., \& Suprun, A. P. (2017). Metodologicheskie peresecheniya psikhosemantiki soznaniya i kvantovoi fiziki [Methodological crossroads of psychosemantics of consciousness and quantum physics]. Moscow: Nestor-Istoriya. (in Russian)

Pezdek, K., \& Salim, R. (2011). Physiological, psychological and behavioral consequences of activating autobiographical memories. Journal of Experimental Social Psychology, 47, 1214-1218.

Platonov, K. I. (1957). Slovo kak fiziologicheskii i lechebnyi faktor [Word as a physiological and curative factor] (2nd ed., revised). Moscow: Medgiz. (in Russian)

Reay, R., Stuart, S., \& Owen, C. (2003). Implementation and effectiveness of interpersonal psychotherapy in a community mental health service. Australasian Psychiatry, 11, 284-289. doi:10.1046/j.1440-1665.2003.00574.x

Roediger, E., \& Dieckmann, E. (2012). Schematherapie: Ein verhaltenstherapeutisches Konzept zur Behandlung verschiedener Persцnlichkeitsstцrungen [Schema therapy: an integrative approach for personality disorders]. Psychotherapie, Psychosomatik, Medizinische Psychologie, 62, 142-148. doi:10.1055/s-0032-1304615 (in German)

Safran, J. D., \& Muran, J. C. (2000). Negotiating the therapeutic alliance: A relational treatment guide. New York: Guilford Press

Safran, J. D., Muran, J. C., DeMaria, A., Boutwell, C., EubanksCarter, C., \& Winston, A. (2014). Investigating the impact of alliance-focused training on interpersonal process and therapists' capacity for experiential reflection. Psychotherapy Research, 24, 269-285. doi:10.1080/10503307.2013.874054

Safran, J. D., \& Segal, Z. V. (1990). Interpersonal process in cognitive therapy. Lanham, MD: Jason Aronson. Sloane, R. B., Staples, F. R., Cristol, A. H., Yorkston, N. J., \& Whipple, K. (1975). Psychotherapy versus behavior therapy. Cambridge, MA: Harvard University Press.

Slobodchikov, V. I. (2004). O perspektivakh postroeniya khristianski orientirovannoi psikhologii [On the perspectives of building the Christianity-oriented psychology]. Moskovskii Psikhoterapevticheskii Zhurnal. Special issue on Christian Psychology, 4, 5-18. (in Russian)

Smith, M. L., Glass, G. V., \& Miller, T. I. (1980). The benefits of psychotherapy. Baltimore, MD: Johns Hopkins University Press. 
Stangier, U., Schramm, E., Heidenreich, T., Berger, M., \& Clark, D. M. (2011). Cognitive therapy vs interpersonal psychotherapy in social anxiety disorder: a randomized controlled trial. Archives of General Psychiatry, 68, 692-700. doi:10.1001/archgenpsychiatry.2011.67

Stiles, W. B., Shapiro, D. A., \& Elliott, R. (1986). Are all psychotherapies equivalent? American Psychologist, 41, 165-180.

Stricker, G., \& Gold, J. R. (2001). An introduction to psychotherapy integration. Psychiatric Times, 18(7). Retrieved from http://www.psychiatrictimes.com/articles/introduction-psychotherapy-integration

Stricker, G., \& Gold, J. (2003). Integrative approaches to psychotherapy. In A. S. Gurman \& S. B. Messer (Eds.), Essential psychotherapies: Theory and practice (2nd ed., pp. 317-349). New York: Guilford Press.

Tasca, G. A., Sylvestre, J., Balfour, L., Chyurlia, L., Evans, J., Fortin-Langelier, B., ... Wilson, B. (2015). What clinicians want: findings from a psychotherapy practice research network survey. Psychotherapy (Chicago, IL), 52(1), 1-11. doi:10.1037/a0038252

Vasilyuk, F. E. (2004). Ispoved' i psikhoterapiya [Confession and psychotherapy]. Moskovskii Psikhoterapevticheskii Zhurnal. Special issue on Christian Psychology, 4, 79-90. (in Russian)

Wachtel, P. L. (1977). Psychoanalysis and behavior therapy: Toward an integration. New York: Basic Books. Wampold, B. E., \& Imel, Z. E. (2015). The great psychotherapy debate: The evidence for what makes psychotherapy work. London: Routledge.

Winter, D. (1990). Therapeutic alternatives for psychological disorders: Personal construct investigations in a health service setting. In G. J. Neimeyer \& R. A. Neimeyer (Eds.), Advances in personal construct theory (Vol. 1, pp. 89-116). Greenwich, CT: JAI Press.

Winter, D. (1992). Personal construct psychology and clinical practice. London: Routledge.

Zarbo, C., Tasca, G. A., Cattafi, F., \& Compare, A. (2016). Integrative psychotherapy works. Frontiers in Psychology, 6, 2021. doi:10.3389/fpsyg.2015.02021

Daria A. Vasilenko - counseling psychologist, PhD in Psychology.

Research Area: personality psychology, psychotherapy, altered states of consciousness.

E-mail: Baskun4ak@mail.ru

Vladimir V. Kucherenko - Senior Research Fellow, Department of Psychology, Lomonosov Moscow State University, PhD in Psychology.

Research Area: personality psychology, hypnotherapy.

E-mail: vvkucherenko@gmail.com

Viktor F. Petrenko - Head of the Laboratory "Communication and psychosemantics", Department of Psychology, Lomonosov Moscow State University, Member of the Russian Academy of Sciences, Professor.

Research Area: general psychology, psychosemantics, theory of consciousness and unconsciousness.

E-mail: victor-petrenko@mail.ru 